\title{
Integrin Subtypes and Nanoscale Ligand Presentation Influence Drug Sensitivity in Cancer Cells
}

\author{
Jennifer L. Young, Ximeng Hua, Heidi Somsel, Florian Reichart, Horst Kessler, and Joachim P. Spatz*
}

Cite This: Nano Lett. 2020, 20, 1183-1191

Read Online

ABSTRACT: Cancer cell-matrix interactions have been shown to enhance cancer cell survival via the activation of pro-survival signaling pathways. These pathways are initiated at the site of interaction, i.e., integrins, and thus, their inhibition has been the target of therapeutic strategies. Individual roles for fibronectinbinding integrin subtypes $\alpha_{\mathrm{v}} \beta_{3}$ and $\alpha_{5} \beta_{1}$ have been shown for various cellular processes; however, a systematic comparison of their function in adhesion-dependent chemoresistance is lacking. Here, we utilize integrin subtype-specific peptidomimetics for $\alpha_{\mathrm{v}} \beta_{3}$ and $\alpha_{5} \beta_{1}$, both as blocking agents on fibronectin-coated surfaces and as surface-immobilized adhesion sites, in order to parse out their role in

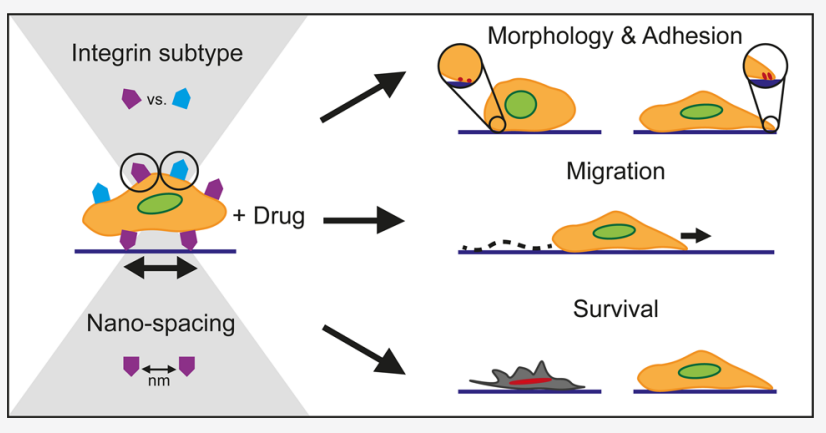
breast cancer cell survival. Block copolymer micelle nanolithography is utilized to immobilize peptidomimetics onto highly ordered gold nanoparticle arrays with biologically relevant interparticle spacings $(35,50$, or $70 \mathrm{~nm})$, thereby providing a platform for ascertaining the dependence of ligand spacing in chemoprotection. We show that several cellular properties-morphology, focal adhesion formation, and migration-are intricately linked to both the integrin subtype and their nanospacing. Importantly, we show that chemotherapeutic drug sensitivity is highly dependent on both parameters, with smaller ligand spacing generally hindering survival. Furthermore, we identify ligand type-specific patterns of drug sensitivity, with enhanced chemosurvival when cells engage $\alpha_{\mathrm{v}} \beta_{3}$ vs $\alpha_{5} \beta_{1}$ on fibronectin; however, this is heavily reliant on nanoscale spacing, as the opposite is observed when ligands are spaced at $70 \mathrm{~nm}$. These data imply that even nanoscale alterations in extracellular matrix properties have profound effects on cancer cell survival and can thus inform future therapies and drug testing platforms.

KEYWORDS: Integrin, extracellular matrix, nanoparticle, peptidomimetic, chemoresistance

$\mathrm{C}$ ells adhere to the extracellular matrix (ECM) via specific receptors, mainly integrins, which subsequently activate signaling pathways that regulate a variety of cellular functions, e.g., motility, proliferation, polarity, differentiation, and survival. ${ }^{1,2}$ In the tumor microenvironment, healthy ECM becomes deregulated, causing changes in both composition and architecture, which cancer cells have been shown to utilize to their advantage. ${ }^{3-5}$ Interactions with the ECM can promote tumor cell extravasation, metastasis, invasion, and most importantly, survival post-chemotherapeutic intervention via the activation of various pro-survival signaling pathways. ${ }^{3,6-10}$ Consequently, researchers have taken two main approaches to mitigating such adhesion-conferred chemoprotection-either via the perturbation of activated pro-survival signaling pathways $^{11-13}$ or via blocking specific integrin-mediated adhesions in order to prevent the onset of signaling pathway initiation. ${ }^{14-16}$ A seminal example of the latter approach, which has also been extensively studied in the clinic, is the blocking of the RGD cell binding sequence via an engineered cyclic RGD pentapeptide, cilengitide (Merck KGaA). Cilengitide has been shown to greatly hinder angiogenesis, thereby starving the tumor and improving subsequent treatment efficacy and patient outcome in specific cases. ${ }^{16-18}$

Despite the exploration of numerous other integrin-targeting strategies (reviewed by Holle et al.) ${ }^{19}$ with varied outcomes, none have advanced into clinic practice. The primary obstacle in interpreting the limited data available on adhesiondependent chemoresistance arises from the enormous molecular complexity of the extracellular environment, thereby making it difficult to identify the specific epitopes that are responsible for protective effects. Moreover, recent studies have shown that adhesion-mediated signaling is affected not only by the chemical nature of the adhesive environment but also by multiple physical features of the matrix, including the spatial patterning of adhesive epitopes available for cell

Received: November 8, 2019

Revised: December 9, 2019

Published: January 7, 2020 


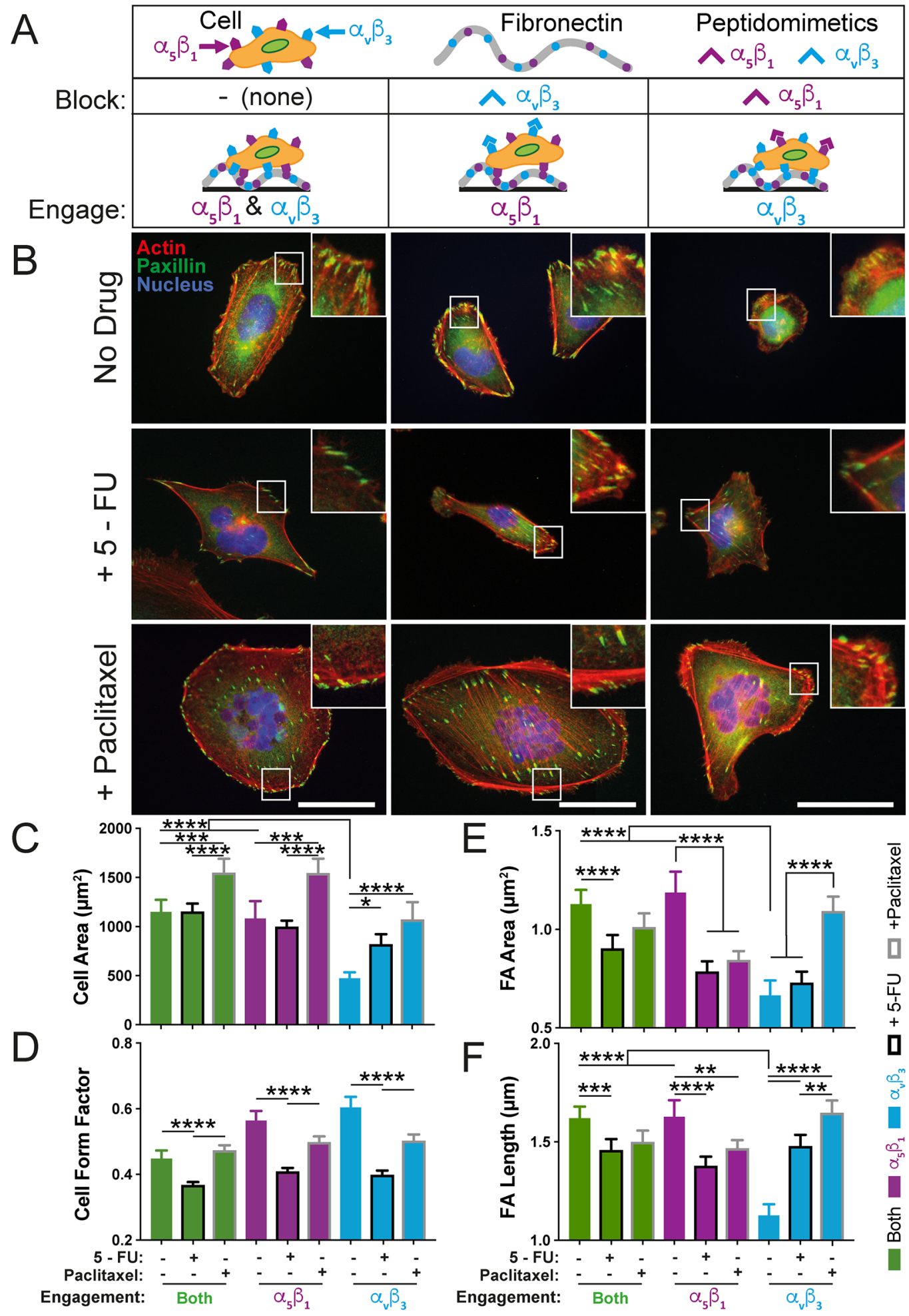

Figure 1. Integrin-specific engagement on fibronectin alters cellular and focal adhesion morphology. (A) MDA-MB-231s were plated on immobilized fibronectin and treated with blocking peptidomimetics as follows: no blocking peptidomimetics engages both integrins [left]; blocking of $\alpha_{\mathrm{v}} \beta_{3}$ (blue $^{\wedge}$ ) results in engagement of $\alpha_{5} \beta_{1}$ (purple) [middle]; and blocking of $\alpha_{5} \beta_{1}$ (purple ${ }^{\wedge}$ ) results in engagement of $\alpha_{\mathrm{v}} \beta_{3}$ (blue) [right]. (B) Cells were stained for actin (red), paxillin (green), and nucleus (blue) for (1) both $\alpha_{5} \beta_{1}$ and $\alpha_{\mathrm{v}} \beta_{3}$ engagement (left column), (2) $\alpha_{5} \beta_{1}$ engagement (middle column), and (3) $\alpha_{\mathrm{v}} \beta_{3}$ engagement (right column), with or without drug treatment (no drug, top row; +5 -FU, middle row; + paclitaxel, bottom row). Insets show zoomed in focal adhesions. Scale bar: $50 \mu \mathrm{m}$. All images without a scale bar have the same scaling as the bottom right image. Cell morphology in terms of (C) cell area (in $\mu \mathrm{m}^{2}$ ) and (D) form factor was quantified for all conditions, i.e., $\alpha_{5} \beta_{1}$ and $\alpha_{\mathrm{v}} \beta_{3}$ engagement ("Both", green bars), $\alpha_{5} \beta_{1}$ engagement (purple bars), and $\alpha_{v} \beta_{3}$ engagement (blue bars), with or without drug treatment as indicated (no drug, no outline; +5-FU, black outline; +paclitaxel, gray outline). Focal adhesion (FA) morphology in terms of (E) FA area (in $\left.\mu \mathrm{m}^{2}\right)$ and (F) major axis length (in $\mu \mathrm{m}$ ) was quantified for all conditions and graphed as in parts C and D. Data are mean $\pm 95 \%$ CI from $n_{\text {cells }}>186$ and $n_{\mathrm{FAs}}>771$. ${ }^{*} p<$ $0.05, * * p<0.01, * * * p<0.001, * * * * p<0.0001$ by one-way ANOVA. All significance comparisons are listed in Table S1. 
binding, the topography of the substrate, and the rigidity of the matrix. 20

Numerous previous reports have identified specific integrin subtypes that contribute to tumor progression (reviewed by Seguin et al.), ${ }^{21}$ including $\alpha_{\mathrm{v}} \beta_{3}$ 's role in breast tumor metastasis to the bone ${ }^{22}$ and $\alpha_{5} \beta_{1}$ 's role in breast cancer cell invasiveness. $^{23}$ While various integrins shown to affect chemosurvival have been the target of therapeutic interventions, a direct comparison of different integrin subtypes in a highly controlled system has not yet been made. Fibronectinbinding integrin subtypes $\alpha_{\mathrm{v}} \beta_{3}$ and $\alpha_{5} \beta_{1}$ exhibit individual roles for various cellular processes including force sensing, mediating structural adaptation to forces, focal adhesion formation, and migration. ${ }^{24-27}$ Most importantly, they have also been shown to regulate chemoresistance in a variety of cancer cells, ${ }^{9}$ which leads us to believe that they could be sensitive to alterations in matrix properties.

When studying cell-ligand interactions, it would be prudent to consider the nanoscale spacing between ligands that are presented to cells. Previous work has shown that cells can indeed sense and differentially respond to nanometer-scale alterations in ligand spacing, affecting their migration, morphology, focal adhesion assembly, cell adhesion, and traction force generation. ${ }^{28-32}$ The spatial organization of available ligand binding partners has been shown to mediate ligand clustering, thereby affecting force-mediated contractility of the cell via "molecular clutches" between integrins and the ECM. ${ }^{31,33,34}$ Most important to this study, nanoscale ligand spacing has been shown to affect melanoma cancer cell behavior and plasticity, ${ }^{35,36}$ yet the degree to which nanoscale ligand spacing affects chemosurvival in cancer cells is still unknown.

While the exact distribution of ligands in physiological tissues is difficult to discern due to limitations in the resolution and accessibility of current imaging techniques, researchers have been able to shed some light on the range of physiologically relevant ligand spacing of proteins and focal complexes within cells using approaches such as immunogold labeling, ${ }^{37}$ crystallography, ${ }^{38,39}$ and advanced imaging techniques. $^{40,41}$ For instance, collagen bundles exhibit a periodic structure occurring around $70 \mathrm{~nm}$ which is believed to be the length scale at which cells attach to the protein, ${ }^{42}$ while periodic binding domains in fibronectin have been identified to be as close as $42 \mathrm{~nm} .^{37}$ Furthermore, heterotypic protein assemblies have been shown to alter binding site spacing, decreasing the distance between sites by up to $20 \%$ from that of homotypic fibrils. ${ }^{37}$ While in vivo measurements are still lacking, these investigations are complicated by the fact that cancer cells can actively reorganize the ECM, likely altering the density of ligand binding sites dynamically. ${ }^{43,44}$ Taken together, we aim to better understand the interplay of integrin subtypes and nanoscale spacing of ligands in the chemosurvival of breast cancer cells by utilizing a highly defined platform.

To achieve this, we investigated two integrin subtypes that are known to be expressed in solid tumors, $\alpha_{\mathrm{v}} \beta_{3}$ and $\alpha_{5} \beta_{1}{ }^{21,45}$ These integrin subtypes recognize the RGD-binding sequence found in proteins commonly present or overexpressed in the tumor microenvironment, including fibronectin, osteopontin, and/or vitronectin. In order to study their individual roles, highly specific integrin subtype peptidomimetics were synthesized. ${ }^{46}$ We first plated human metastatic MDA-MB231 breast cancer cells on human fibronectin (Fn), which contains binding sites for both $\alpha_{\mathrm{v}} \beta_{3}$ and $\alpha_{5} \beta_{1}$ integrin subtypes, and examined cell morphology with or without the addition of subtype-specific peptidomimetics capable of blocking integrins $\alpha_{\mathrm{v}} \beta_{3}$ or $\alpha_{5} \beta_{1}$ (experimental design outlined in Figure 1A). When blocking $\alpha_{\mathrm{v}} \beta_{3}$ (thereby engaging $\alpha_{5} \beta_{1}$ ), cells maintain a similar spread area compared to Fn alone, and become slightly more rounded (Figure 1B, top row; Figure 1C). When blocking $\alpha_{5} \beta_{1}$, thereby engaging $\alpha_{\mathrm{v}} \beta_{3}$, cells become significantly smaller and rounder (Figure 1B, top row; Figure 1C,D), which is consistent with cells plated on vitronectin $(\mathrm{Vn})$, where $\alpha_{\mathrm{v}} \beta_{3}$ is the major binding integrin (Figure S1A-C).

In order to assess effects of chemotherapeutic drugs, we employed two commonly used compounds from which cells have been reported to have developed chemoresistance: 5fluorouracil (5-FU), which blocks DNA replication, and paclitaxel (also commonly referred to as Taxol), which disrupts microtubule breakdown. ${ }^{47}$ When treated with 5-FU, cells on Fn become more elongated in all conditions (Figure 1B, middle row; Figure 1D), while paclitaxel treatment causes cells to become very large and rounded (Figure 1B, bottom row; Figure $1 \mathrm{C}, \mathrm{D})$, highlighting that the different mechanisms of action of the two drugs can affect subsequent cell morphology in surviving cells. The fact that these responses are also dependent upon specific ligand interactions underscores the synergy between these two biochemical pathways (Figure 1B).

Focal adhesion (FA) formation and the subsequent activation of downstream signaling pathways have been shown to be important indicators of cancer cell invasiveness. ${ }^{48}$ As FAs play a major role in substrate sensing and mechanotransduction, ${ }^{49}$ previous studies have linked alterations in FA formation to various substrate properties, e.g., stiffness, ligand type, topography, dimensionality, etc. ${ }^{50,51}$ Our results show that FA formation is affected not only by integrin subtype engagement but also by drug treatment. First, we observe that cells engaging only $\alpha_{\mathrm{v}} \beta_{3}$ form small, short adhesions (Figure 1B, top row; Figure 1E,F; Figure S1A,D,E). While drug treatment does not greatly affect FA formation on Fn alone, engagement of $\alpha_{5} \beta_{1}$ greatly hinders FA clustering in drug-treated cells (Figure 1B, middle and bottom rows; Figure $1 \mathrm{E}, \mathrm{F})$. Conversely, drug treatment in conjunction with $\alpha_{\mathrm{v}} \beta_{3}$ engagement results in FA size enhancement, with paclitaxel inducing the largest FAs (Figure 1B, middle and bottom rows; Figure 1E,F). Overall, both cell and FA morphology were most notably affected by $\alpha_{\mathrm{v}} \beta_{3}$ engagement, further underscoring the role this integrin subtype plays in breast cancer cell behavior.

While native proteins are an important substrate for studying integrin subtype-specific effects, uncontrolled parameters are inherent to such systems (e.g., ligand spacing, the presence of other integrin subtype binding sites, etc.) that can obscure the role of primary mediators of cell-ECM behavior. To better isolate these variables, we synthesized highly ordered gold nanoparticle (AuNP) arrays via the previously described block copolymer micelle nanolithography (BCMN) technique ${ }^{52}$ in order to control both ligand spacing/density and type. AuNP arrays with interparticle spacings of 35,50 , and $70 \mathrm{~nm}$ were produced and characterized via SEM (Figure S3). Such biologically relevant spacings were chosen based on previous reports of protein binding site organization, conformation of proteins, and in vitro experiments showing restricted integrin clustering on substrates with ligand spacing greater than $\sim 73$ $n .^{20}$ Thiolated versions of the aforementioned integrinsubtype-specific peptidomimetics were synthesized that could specifically attach to the AuNPs, ${ }^{46}$ thereby resulting in a highly 


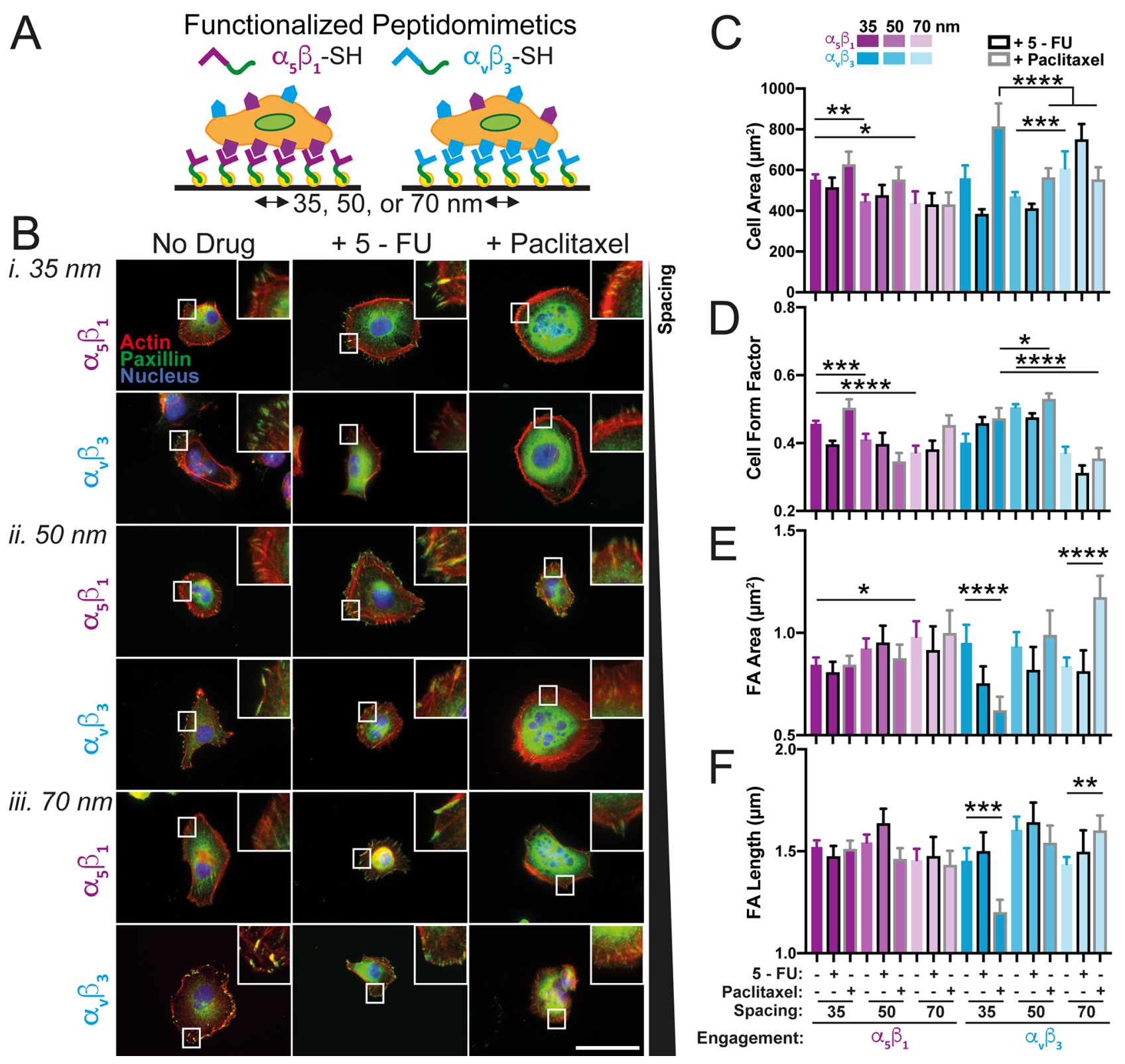

Figure 2. Integrin subtype-specific engagement at defined nanospacing alters cellular and focal adhesion morphology. (A) MDA-MB-231s were plated on integrin-specific peptidomimetics, i.e., $\alpha_{5} \beta_{1}$ (purple) or $\alpha_{\mathrm{v}} \beta_{3}$ (blue), immobilized on AuNPs with interspacings of 35, 50, or 70 nm. (B) Cells were stained for actin (red), paxillin (green), and nucleus (blue) on samples with AuNP spacing of (i) $35 \mathrm{~nm}$, (ii) $50 \mathrm{~nm}$, or (iii) $70 \mathrm{~nm}$ functionalized with either $\alpha_{5} \beta_{1}$ (top of each set) or $\alpha_{v} \beta_{3}$ (bottom of each set), with or without drug treatment (no drug, left column; +5 -FU, middle column; +paclitaxel, right column). Insets show zoomed in focal adhesions. Scale bar: $50 \mu \mathrm{m}$. (C) Cell morphology in terms of area $\left(\mathrm{C}, \mu \mathrm{m}^{2}\right)$ and form factor (D) was quantified for all conditions, i.e., $\alpha_{5} \beta_{1}$ (purple) or $\alpha_{\mathrm{v}} \beta_{3}$ (blue) engagement at 35, 50, or $70 \mathrm{~nm}$ (where decreasing opacity corresponds with increasing spacing), with or without drug treatment (no drug, no outlines; +5-FU, black outline; +paclitaxel, gray outline). Focal adhesion (FA) morphology in terms of area $\left(\mathrm{E}, \mu \mathrm{m}^{2}\right)$ and major axis length $(\mathrm{F}, \mu \mathrm{m})$ was quantified and graphed for all conditions as in parts $\mathrm{C}$ and D. Data are mean $\pm 95 \% \mathrm{CI}$ from $n_{\text {cells }}>71$ and $n_{\mathrm{FAs}}>376$. $*_{p}<0.05, * * p<0.01, * * * p<0.001, * * * * p 0.0001$ by one-way ANOVA. All significance comparisons are listed in Table S2.

controlled and highly defined nanoscale substrate (schematic of experimental outline in Figure 2A).

In general, cells were smaller on AuNP surfaces compared to Fn-coated substrates (Figure 2C vs Figure 1C), likely due to differences in binding site availability and organization, which would be enhanced on Fn-coated substrates. Compared to cells on $\alpha_{5} \beta_{1}$-coated surfaces, cells on $\alpha_{\mathrm{v}} \beta_{3}$-coated surfaces were much larger and more fully spread on $70 \mathrm{~nm}$ spacing (Figure 2B-D). Overall, both integrin subtypes were affected by spacing, albeit at different length scales, as previously reported for various other cell types. ${ }^{29,53}$ On $\alpha_{5} \beta_{1}$, cell spread area and form factor were significantly higher on $35 \mathrm{~nm}$ compared to 50 or $70 \mathrm{~nm}$, while on $\alpha_{\mathrm{v}} \beta_{3}$, cells on $50 \mathrm{~nm}$ spacing elicited a significantly different morphology compared to $70 \mathrm{~nm}$ (Figure 2C,D). Drug treatment was found to enhance this spacing-sensitive response. Paclitaxel-treated cells plated on $\alpha_{\mathrm{v}} \beta_{3}$ exhibited significant differences in cell area between 35 and 50 or $70 \mathrm{~nm}$ (Figure 2C). When comparing the effects of specific spacings on the two integrin subtypes, larger nanoscale ligand spacing resulted in the greatest changes between the two, regardless of condition, while cells on $35 \mathrm{~nm}$ were more highly affected by drug treatment on $\alpha_{\mathrm{v}} \beta_{3}$ vs $\alpha_{5} \beta_{1}$ 
A

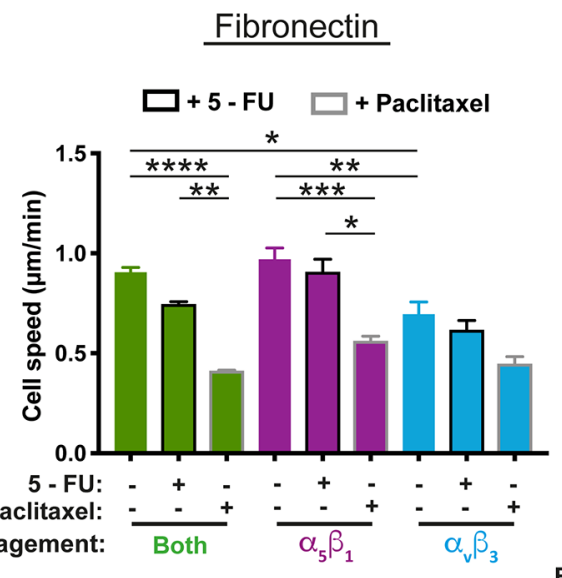

B

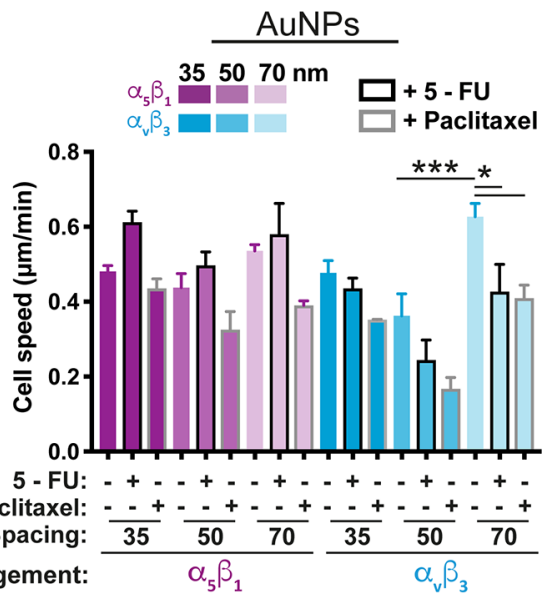

Engagement:

C Engagement:

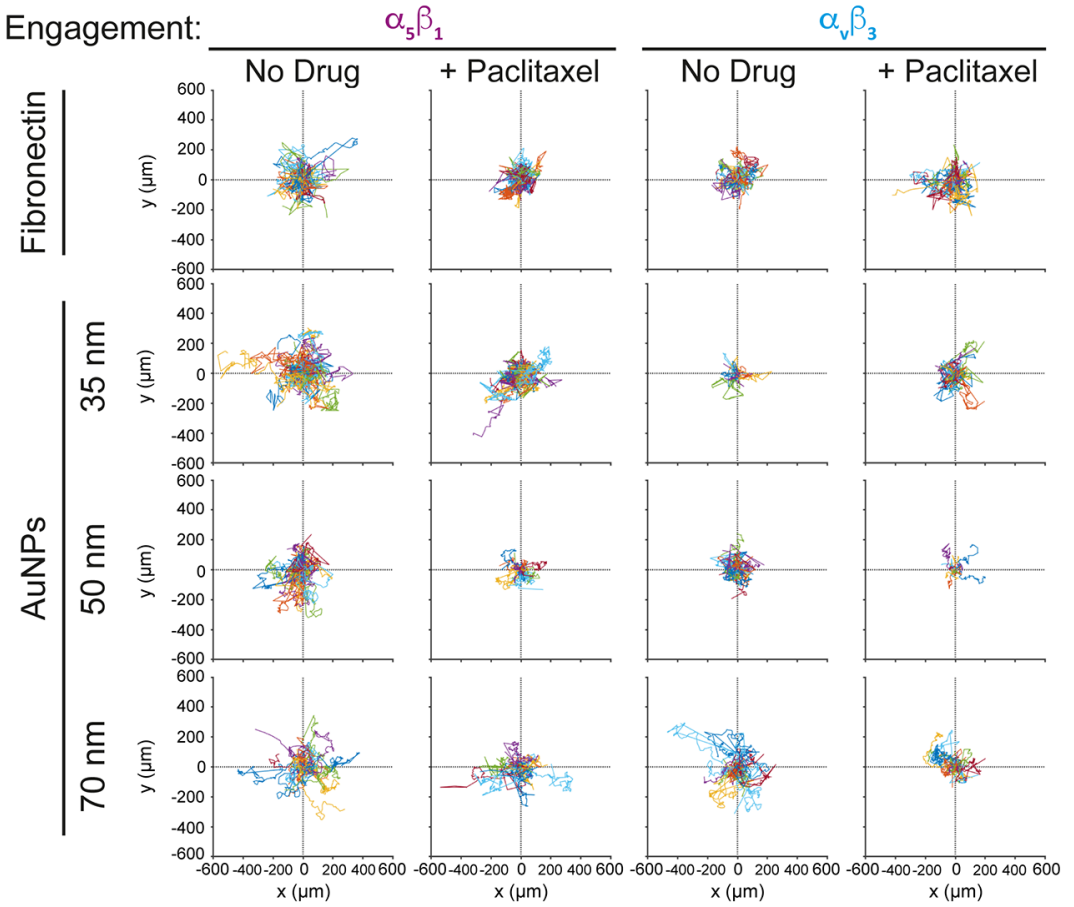

Figure 3. Integrin subtype-specific and nanospacing-specific cell motility. (A) Cell motility (cell speed in $\mu \mathrm{m} / \mathrm{min}$ ) for integrin subtype-specific engagement on fibronectin using blocking peptidomimetics vs ligand type (both $\alpha_{5} \beta_{1}$ and $\alpha_{v} \beta_{3}$ engagement, green; $\alpha_{5} \beta_{1}$ engagement, purple; $\alpha_{\mathrm{v}} \beta_{3}$ engagement, blue) and drug treatment (no drug, no outline; +5-FU, black outline; +paclitaxel, gray outline). (B) Cell motility for integrin subtypespecific engagement on peptidomimetics immobilized on AuNPs with interspacings of 35, 50, or $70 \mathrm{~nm}$. Data plotted as in Figure 2, i.e., $\alpha_{5} \beta_{1}$ (purple) or $\alpha_{v} \beta_{3}$ (blue) engagement at 35, 50, or $70 \mathrm{~nm}$ (where decreasing opacity corresponds with increasing spacing), with or without drug treatment (no drug, no outlines; +5-FU, black outline; +paclitaxel, gray outline). (C) Representative cell trajectories depicted as roseplots from the first $17 \mathrm{~h}$ of the experiment of cells engaging $\alpha_{5} \beta_{1}$ (two left columns) or $\alpha_{v} \beta_{3}$ (two right columns) without drug treatment or with paclitaxel treatment (left and right column, respectively, of each set) for cells plated on fibronectin (top row) or on AuNPs with interspacing of $35 \mathrm{~nm}$ (second row), $50 \mathrm{~nm}$ (third row), and $70 \mathrm{~nm}$ (last row). $n_{\mathrm{Fn}, \text { tracks }}>287 ; n_{\mathrm{AuNP}, \text { tracks }}>93 ; r=2$. Data are mean $\pm \mathrm{SEM}$. $* p<0.05, * * p<0.01, * * * p$ $<0.001, * * * * p<0.0001$ by one-way ANOVA.

(Figure 2C,D). Additionally, cells plated on $\alpha_{\mathrm{v}} \beta_{3}$-coated surfaces were more affected by drug treatment than those on $\alpha_{5} \beta_{1}$, exhibiting a decrease in cell area with 5-FU treatment at 35 and $50 \mathrm{~nm}$, and an increase at $70 \mathrm{~nm}$. The exact opposite of this pattern was observed in response to paclitaxel treatment, suggesting that the nanoscale environment can impact the morphological response to chemo drugs in a pathway-specific fashion (Figure 2B,C).

Mirroring morphological observations, FA formation in cells on $\alpha_{\mathrm{v}} \beta_{3}$-coated surfaces was more affected by drug treatment than FAs in cells on $\alpha_{5} \beta_{1}$-coated surfaces (Figure 2B,E,F). FA area, but not necessarily length, on $\alpha_{5} \beta_{1}$ is generally enhanced on larger spacing vs smaller spacing (Figure 2B,E,F), as has been previously reported in osteosarcoma cells. ${ }^{53}$ Interestingly, on $\alpha_{\mathrm{v}} \beta_{3}$-coated surfaces, FA area and length were particularly sensitive to paclitaxel treatment. Compared to control groups, FA area and length in paclitaxel-treated cells decreased on 35 $\mathrm{nm}$, remained unchanged on $50 \mathrm{~nm}$, and increased on $70 \mathrm{~nm}$ (Figure 2E,F). In general, drug treatment significantly altered FA characteristics in almost all conditions. This shows that, while neither drug specifically targets focal contacts, global changes in cellular organization in response to chemo drugs are connected to dynamic alterations of focal adhesion presentation, and that these alterations are modulated by nanoscale 

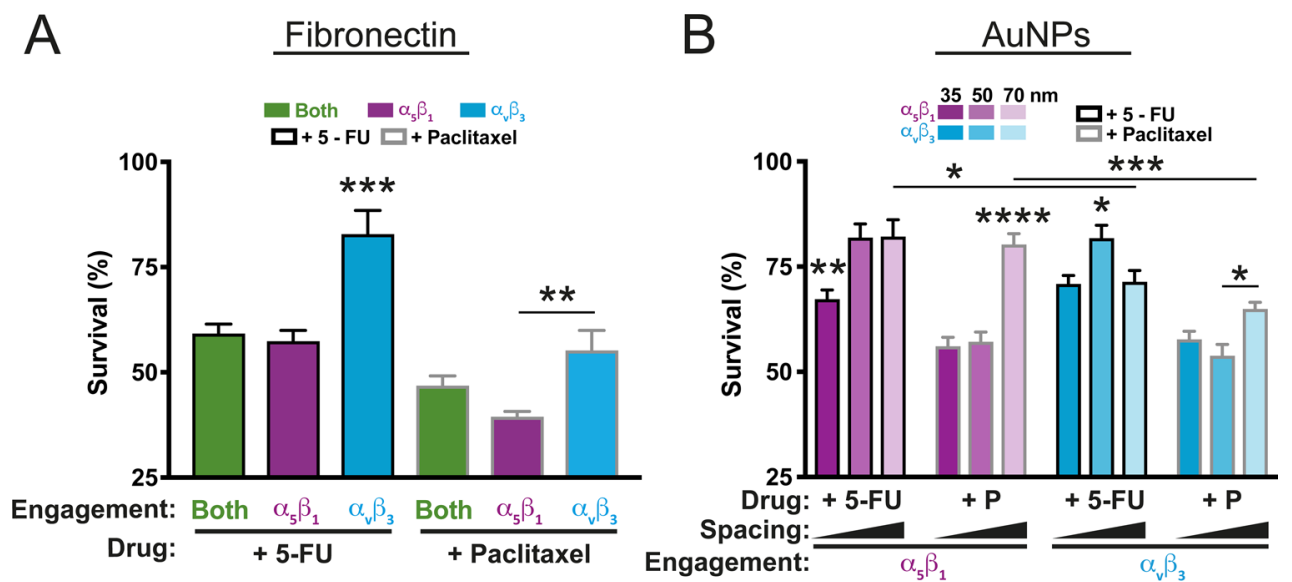

Figure 4. Chemosurvival is dependent on integrin subtypes and nanoscale dimensionality. (A) Percent survival of cells treated with 5-FU (black outline) or paclitaxel (gray outline) plated on fibronectin with integrin-specific engagement as indicated (both, i.e., $\alpha_{5} \beta_{1}$ and $\alpha_{v} \beta_{3}$, green; $\alpha_{5} \beta_{1}$, purple; $\alpha_{\mathrm{v}} \beta_{3}$, blue). (B) Percent survival of cells treated with 5-FU (black outline) or paclitaxel (denoted as "P" on the $x$-axis, gray outline) on AuNPs functionalized with integrin-subtype-specific peptidomimetics $\left(\alpha_{5} \beta_{1}\right.$ engagement, purple; $\alpha_{\mathrm{v}} \beta_{3}$ engagement, blue) at interspacing of 35, 50, or $70 \mathrm{~nm}$ (where decreasing opacity corresponds with increasing spacing). For samples in part A, $n=8$; part B, $n=7$. Data are mean \pm SEM. ** $p<$ $0.01, * * * p<0.001$ by one-way ANOVA for part A and $* p<0.05, * * p<0.01, * * * p<0.001, * * * *<0.0001$ by two-way ANOVA for part B.

ECM organization. Taken together, cellular and FA morphology data indicate that cancer cells are sensitive to both ligand type and nanoscale ligand spacing, and that this sensitivity may play a role in ECM-modulated chemoresistance.

Altered cell motility usually accompanies the transformation of a cancer cell to a metastatic or chemoresistant phenotype. ${ }^{54,55}$ Additionally, subtype-specific integrin expression and abundance have been shown to be altered in cancer progression. ${ }^{56}$ Therefore, we analyzed cancer cell migration as a function of integrin engagement, dimensionality, and drug treatment (Figure 3). We found that cells plated on Fn slowed in response to either drug treatment, but most significantly with paclitaxel. Cells engaging only $\alpha_{5} \beta_{1}$ on $\mathrm{Fn}$ behaved similarly to cells in which both integrins could be bound (i.e., on Fn alone), mirroring cell morphology patterns (Figure 1). When engaging only $\alpha_{\mathrm{v}} \beta_{3}$ on Fn, cell speed decreased, a pattern which was enhanced with paclitaxel treatment (Figure 3A). On AuNP arrays, cells on $\alpha_{\mathrm{v}} \beta_{3}$-coated surfaces were slower, in general, than cells on $\alpha_{5} \beta_{1}$-coated surfaces (Figure $3 \mathrm{~B})$. Interestingly, cells of all conditions were slower on $50 \mathrm{~nm}$ spacing than on 35 or $70 \mathrm{~nm}$ spacing, where they exhibited similar speeds. While both drug treatments resulted in decreased cell speed on $\alpha_{\mathrm{v}} \beta_{3}$, only paclitaxel reduced speed on $\alpha_{5} \beta_{1}$. Lastly, cell trajectories on Fn or AuNPs treated with paclitaxel were examined compared to controls due to the fact that paclitaxel treatment hindered cell movement in nearly all conditions (Figure 3C). Cells on $70 \mathrm{~nm}$ spaced AuNPs were more exploratory than those on narrower spacings, which was most pronounced on $\alpha_{\mathrm{v}} \beta_{3}$-coated surfaces. This was echoed on fibronectin-coated substrates, where paclitaxel-treated cells engaging $\alpha_{\mathrm{v}} \beta_{3}$ were also more exploratory than those engaging $\alpha_{5} \beta_{1}$ (Figure 3C). Previous studies have found differing roles for integrin subtypes in migration; e.g., $\beta_{1}$ promotes a more random migration pattern compared to $\beta_{3}$, which is ultimately regulated through Rho GTPases. ${ }^{57}$ These data suggest that this is not only a function of integrin subtype but also the nanoscale presentation of extracellular ligands in combination with drug treatment.

While cell metrics like morphology, focal adhesion formation, and motility show that both integrin subtype and ligand nanospacing are important mediators of cancer cell behavior, how these two parameters influence cell survival has important implications in therapeutic applications and drug screening. Therefore, cell survival was assessed after $48 \mathrm{~h}$ of drug treatment based on integrin subtype on both Fn and AuNP arrays, with either 5-FU or paclitaxel treatment (Figure 4). When treated with either drug, cells on Fn alone exhibited similar survival as when only $\alpha_{5} \beta_{1}$ was engaged on Fn (Figure 4A). However, when only $\alpha_{\mathrm{v}} \beta_{3}$ was engaged on Fn, survival was enhanced by up to $\sim 20 \%$ with 5-FU treatment and $\sim 10 \%$ with paclitaxel (Figure 4A).

The similarities found between the engagement of both integrin subtypes and the $\alpha_{5} \beta_{1}$-only engagement can be explained by the fact that MDA-MB-231 cells express $\beta_{1}$ more readily than $\alpha_{\mathrm{v}}$, ${ }^{58}$ indicating that $\alpha_{5} \beta_{1}$ is likely the dominant ligand partner for MDA-MB-231 cells on Fn. Although, expression alone does not necessarily indicate functional output, as distinct but complementary roles for these two integrin subtypes in regulating contractility and adhesion strength have been shown in fibroblasts. ${ }^{24,59}$ Furthermore, it is well-known that $\alpha_{\mathrm{v}} \beta_{3}$ promotes breast cancer metastasis to the bone niche, ${ }^{22}$ which could explain the enhancement in chemosurvival we observe when engaging only this integrin subtype.

Next, we looked at drug responsiveness as a function of ligand spacing and integrin subtype using the AuNP arrays. Importantly, we observed that cancer cell survival in response to chemotherapeutic treatment is highly dependent on nanoscale ligand spacing, an observation not yet reported in the literature. We demonstrated differences in survival not only between the two integrin subtypes, but also between drug type and ligand spacing. When cancer cells engage $\alpha_{5} \beta_{1}$ integrins, higher spacing improves survival with both 5-FU and paclitaxel treatment, but the length scale at which this occurs is drugdependent; i.e., $50 \mathrm{~nm}$ enhances survival with 5-FU, while larger spacing is required for paclitaxel (Figure 4B). On $\alpha_{\mathrm{v}} \beta_{3}$, we observe the same trend with paclitaxel treatment, but a different trend in how spacing affects cell survival with 5-FU treatment, namely, that $50 \mathrm{~nm}$ spacing enhances chemosurvival (Figure 4B).

Optimal ligand spacing for cell spreading has previously been reported in various cell lines to be between $\sim 58$ and 73 
$\mathrm{nm} .^{28}$ Indeed, we observed that survival was low on $35 \mathrm{~nm}$ substrates vs larger spacing for all conditions, indicating that integrin-mediated pro-survival signaling could be hindered at such length scales. Supporting this, we found impaired focal adhesion formation at $35 \mathrm{~nm}$, as has been previously demonstrated in melanoma cells. In that investigation, the authors linked this impaired FA formation to the restricted size of focal adhesion proteins, i.e., talin at $\sim 60 \mathrm{~nm}$, and the restricted lateral motion of integrins during clustering. ${ }^{35}$

Most notably, we find that, by altering only nanoscale ligand spacing, we were able to increase cancer cell chemosurvival by over $40 \%$ (Figure $4 \mathrm{~B}, \alpha_{5} \beta_{1} /$ paclitaxel $/ 50$ vs $70 \mathrm{~nm}$ ), a surprisingly large magnitude given the small change in ECM conditions. These data suggest that even nanoscale alterations in ligand presentation can significantly alter cancer cell survival in response to chemotherapeutic treatment, and that these alterations are affected by not only the ligand type but also the type of drug utilized in treatment. The observed differential responses in chemosurvival among the different conditions likely stem from integrin subtype-specific activation of prosurvival signaling pathways, e.g., FAK, PI3K, or Akt, ${ }^{60,61}$ which would be worthwhile to explore in future studies.

Although this study examined nanoscale ligand spacing on rigid glass substrates, ECM stiffness has been shown to be an important mediator in mechanotransduction. Moreover, it has been shown that matrix stiffness affects chemoresistance through such mechanisms as cellular stiffening, focal adhesion-driven signaling, or promoting the epithelial-tomesenchymal transition. ${ }^{10,62,63}$ Intriguingly, the $\alpha_{5} \beta_{1}$ and $\alpha_{\mathrm{v}} \beta_{3}$ integrin subtypes have been shown to distinctly modulate force transmission at the cell-ECM interface ${ }^{64}$ via the activation of unique signaling pathways. ${ }^{65-67}$ Thus, future studies will focus on untangling the complex interplay of matrix stiffness and ligand presentation in regulating chemoresistance. This can be realized by utilizing the well-established technique of transfer nanolithography, in which AuNP arrays can be transferred onto soft polymeric substrates. ${ }^{68}$ Taken together, these data call not only for a better understanding of the intricate tumor microenvironment but also for more faithful ECM mimics for cancer drug screening.

\section{ASSOCIATED CONTENT}

\section{SI Supporting Information}

The Supporting Information is available free of charge at https://pubs.acs.org/doi/10.1021/acs.nanolett.9b04607.

Materials and methods section, description of supporting experiments, tables of signficance, and additional data and figures including cells plated on vitronectin, integrin subtype immunostaining, SEM of samples produced by block copolymer micelle nanolithography, cellular proliferation, drug dose curves, and low magnification images of cells (PDF)

\section{AUTHOR INFORMATION}

\section{Corresponding Author}

Joachim P. Spatz - Max Planck Institute for Medical

Research, Heidelberg, Germany, and Heidelberg

University, Heidelberg, Germany; (1) orcid.org/0000-

0003-3419-9807; Phone: +49 6221 486-420;

Email: spatz@mr.mpg.de

\section{Other Authors}

Jennifer L. Young - Max Planck Institute for Medical Research, Heidelberg, Germany, and Heidelberg University, Heidelberg, Germany

Ximeng Hua - Max Planck Institute for Medical Research, Heidelberg, Germany, and Heidelberg University, Heidelberg, Germany

Heidi Somsel - Max Planck Institute for Medical Research, Heidelberg, Germany, and Heidelberg University, Heidelberg, Germany

Florian Reichart - Technical University of Munich, Garching, Germany

Horst Kessler - Technical University of Munich, Garching, Germany; (1) orcid.org/0000-0002-72929789

Complete contact information is available at: https://pubs.acs.org/10.1021/acs.nanolett.9b04607

\section{Author Contributions}

J.L.Y. and J.P.S. designed the research. J.L.Y., X.H., and H.S. performed the research. J.L.Y. and X.H. analyzed the data. F.R. and H.K. synthesized the peptidomimetics. J.L.Y. and J.P.S. wrote the paper.

\section{Notes}

The authors declare no competing financial interest.

\section{ACKNOWLEDGMENTS}

This work was supported by the Max Planck Society, the German Federal Ministry of Education and Research, and the European Research Council (to J.L.Y. and J.P.S.). The authors would like to thank Dr. Andrew Holle, PD Dr. Dr. Elisabetta Ada Cavalcanti-Adam, Dr. Jacopo Di Russo, and Dr. Dimitris Missirlis for experimental discussions, as well as Janis Grigoridis for SEM support. The authors would like to acknowledge funding from the MaxSynBio Consortium, which is jointly funded by the Federal Ministry of Education and Research of Germany and the Max Planck Society. They further acknowledge support from the VolkswagenStiftung (priority call 'Life?'). J.P.S. is the Weston Visiting Professor at the Weizmann Institute of Science and part of the excellence cluster CellNetworks at the University of Heidelberg.

\section{REFERENCES}

(1) Hynes, R. O. The Extracellular Matrix: Not Just Pretty Fibrils. Science 2009, 326, 1216-1219.

(2) Wozniak, M. A.; Modzelewska, K.; Kwong, L.; Keely, P. J. Focal Adhesion Regulation of Cell Behavior. Biochim. Biophys. Acta, Mol. Cell Res. 2004, 1692, 103-119.

(3) Oskarsson, T. Extracellular Matrix Components in Breast Cancer Progression and Metastasis. Breast 2013, 22, S66-S72.

(4) Larsen, M.; Artym, V. V.; Green, J. A.; Yamada, K. M. The Matrix Reorganized: Extracellular Matrix Remodeling and Integrin Signaling. Curr. Opin. Cell Biol. 2006, 18, 463-471.

(5) Allinen, M.; Beroukhim, R.; Cai, L.; Brennan, C.; LahtiDomenici, J.; Huang, H.; Porter, D.; Hu, M.; Chin, L.; Richardson, A. Molecular Characterization of the Tumor Microenvironment in Breast Cancer. Cancer Cell 2004, 6, 17-32.

(6) Glinsky, G. V. Anti-Adhesion Cancer Therapy. Cancer Metastasis Rev. 1998, 17, 177-185.

(7) Juliano, R. L.; Varner, J. A. Adhesion Molecules in Cancer: the Role of Integrins. Curr. Opin. Cell Biol. 1993, 5, 812-818. 
(8) Aoudjit, F.; Vuori, K. Integrin Signaling Inhibits PaclitaxelInduced Apoptosis in Breast Cancer Cells. Oncogene 2001, 20, 49955004.

(9) Aoudjit, F.; Vuori, K. Integrin Signaling in Cancer Cell Survival and Chemoresistance. Chemother. Res. Pract. 2012, 2012, 1-16.

(10) Joyce, M. H.; Lu, C.; James, E. R.; Hegab, R.; Allen, S. C.; Suggs, L. J.; Brock, A. Phenotypic Basis for Matrix StiffnessDependent Chemoresistance of Breast Cancer Cells to Doxorubicin. Front. Oncol. 2018, 8, 57.

(11) Muranen, T.; Selfors, L. M.; Worster, D. T.; Iwanicki, M. P.; Song, L.; Morales, F. C.; Gao, S.; Mills, G. B.; Brugge, J. S. Inhibition of PI3K/mTOR Leads to Adaptive Resistance in Matrix-Attached Cancer Cells. Cancer Cell 2012, 21, 227-239.

(12) Baselga, J. Targeting the Phosphoinositide-3 (PI3) Kinase Pathway in Breast Cancer. Oncologist 2011, 16 (S1), 12-19.

(13) Courtney, K. D.; Corcoran, R. B.; Engelman, J. A. The PI3K Pathway as Drug Target in Human Cancer. J. Clin. Oncol. 2010, 28, $1075-1083$

(14) Liu, Z.; Wang, F.; Chen, X. Integrin $\alpha \mathrm{V} \beta 3$-Targeted Cancer Therapy. Drug Dev. Res. 2008, 69, 329-339.

(15) Hersey, P.; Sosman, J.; O’Day, S.; Richards, J.; Bedikian, A.; Gonzalez, R.; Sharfman, W.; Weber, R.; Logan, T.; Buzoianu, M.; et al. A Randomized Phase 2 Study of Etaracizumab, a Monoclonal Antibody Against Integrin $\alpha \mathrm{V} \beta 3, \pm$ Dacarbazine in Patients with Stage IV Metastatic Melanoma. Cancer 2010, 116, 1526-1534.

(16) Stupp, R.; Hegi, M. E.; Gorlia, T.; Erridge, S. C.; Perry, J.; Hong, Y.-K.; Aldape, K. D.; Lhermitte, B.; Pietsch, T.; Grujicic, D.; et al. Cilengitide Combined with Standard Treatment for Patients with Newly Diagnosed Glioblastoma with Methylated MGMT Promoter (CENTRIC EORTC 26071-22072 Study): a Multicentre, Randomised, Open-Label, Phase 3 Trial. Lancet Oncol. 2014, 15, $1100-1108$.

(17) Dechantsreiter, M. A.; Planker, E.; Mathä, B.; Lohof, E.; Hölzemann, G.; Jonczyk, A.; Goodman, S. L.; Kessler, H. NMethylated Cyclic RGD Peptides as Highly Active and Selective $\alpha \mathrm{V} \beta 3$ Integrin Antagonists. J. Med. Chem. 1999, 42, 3033-3040.

(18) Mas-Moruno, C.; Rechenmacher, F.; Kessler, H. Cilengitide: the First Anti-Angiogenic Small Molecule Drug Candidate. Design, Synthesis and Clinical Evaluation. Anti-Cancer Agents Med. Chem. 2010, 10, 753-768.

(19) Holle, A. W.; Young, J. L.; Spatz, J. P. In Vitro Cancer CellECM Interactions Inform In Vivo Cancer Treatment. Adv. Drug Delivery Rev. 2016, 97, 270-279.

(20) Young, J. L.; Holle, A. W.; Spatz, J. P. Nanoscale and Mechanical Properties of the Physiological Cell-ECM Microenvironment. Exp. Cell Res. 2016, 343, 3-6.

(21) Seguin, L.; Desgrosellier, J. S.; Weis, S. M.; Cheresh, D. A. Integrins and Cancer: Regulators of Cancer Stemness, Metastasis, and Drug Resistance. Trends Cell Biol. 2015, 25, 234-240.

(22) Sloan, E. K.; Pouliot, N.; Stanley, K. L.; Chia, J.; Moseley, J. M.; Hards, D. K.; Anderson, R. L. Tumor-Specific Expression of $\alpha \mathrm{v} \beta 3$ Integrin Promotes Spontaneous Metastasis of Breast Cancer to Bone. Breast Cancer Res. 2006, 8, R20.

(23) Mierke, C. T.; Frey, B.; Fellner, M.; Herrmann, M.; Fabry, B. Integrin $\alpha 5 \beta 1$ Facilitates Cancer Cell Invasion Through Enhanced Contractile Forces. J. Cell Sci. 2011, 124, 369-383.

(24) Schiller, H. B.; Hermann, M.-R.; Polleux, J.; Vignaud, T.; Zanivan, S.; Friedel, C. C.; Sun, Z.; Raducanu, A.; Gottschalk, K.-E.; Théry, M.; et al. $\beta_{1}$ - and $\alpha_{\mathrm{V}}$-Class Integrins Cooperate to Regulate Myosin II During Rigidity Sensing of Fibronectin-Based Microenvironments. Nat. Cell Biol. 2013, 15, 625-636.

(25) Ballestrem, C.; Hinz, B.; Imhof, B. A.; Wehrle-Haller, B. Marching at the Front and Dragging Behind: Differential $\alpha \mathrm{V} \beta 3$ Integrin Turnover Regulates Focal Adhesion Behavior. J. Cell Biol. 2001, 155, 1319-1332.

(26) Danen, E. H. J.; Sonneveld, P.; Brakebusch, C.; Fässler, R.; Sonnenberg, A. The Fibronectin-Binding Integrins $\alpha 5 \beta 1$ and $\alpha \mathrm{v} \beta 3$ Differentially Modulate RhoA-GTP Loading, Organization of Cell
Matrix Adhesions, and Fibronectin Fibrillogenesis. J. Cell Biol. 2002, 159, 1071-1086.

(27) White, D. P.; Caswell, P. T.; Norman, J. C. $\alpha \mathrm{v} \beta 3$ and $\alpha 5 \beta 1$ Integrin Recycling Pathways Dictate Downstream Rho Kinase Signaling to Regulate Persistent Cell Migration. J. Cell Biol. 2007, $177,515-525$.

(28) Arnold, M.; Cavalcanti-Adam, E. A.; Glass, R.; Blümmel, J.; Eck, W.; Kantlehner, M.; Kessler, H.; Spatz, J. P. Activation of Integrin Function by Nanopatterned Adhesive Interfaces. ChemPhysChem 2004, 5, 383-388.

(29) Cavalcanti-Adam, E.; Micoulet, A.; Blummel, J.; Auernheimer, J.; Kessler, H.; Spatz, J. Lateral Spacing of Integrin Ligands Influences Cell Spreading and Focal Adhesion Assembly. Eur. J. Cell Biol. 2006, $85,219-224$.

(30) Cavalcanti-Adam, E. A.; Volberg, T.; Micoulet, A.; Kessler, H.; Geiger, B.; Spatz, J. P. Cell Spreading and Focal Adhesion Dynamics Are Regulated by Spacing of Integrin Ligands. Biophys. J. 2007, 92, 2964-2974.

(31) Oria, R.; Wiegand, T.; Escribano, J.; Elosegui-Artola, A.; Uriarte, J. J.; Moreno-Pulido, C.; Platzman, I.; Delcanale, P.; Albertazzi, L.; Navajas, D.; et al. Force Loading Explains Spatial Sensing of Ligands by Cells. Nature 2017, 552, 219.

(32) Selhuber-Unkel, C.; Erdmann, T.; López-García, M.; Kessler, H.; Schwarz, U. S.; Spatz, J. P. Cell Adhesion Strength Is Controlled by Intermolecular Spacing of Adhesion Receptors. Biophys. J. 2010, 98, 543-551.

(33) Irvine, D. J.; Hue, K.-A.; Mayes, A. M.; Griffith, L. G. Simulations of Cell-Surface Integrin Binding to Nanoscale-Clustered Adhesion Ligands. Biophys. J. 2002, 82, 120-132.

(34) Liu, Y.; Medda, R.; Liu, Z.; Galior, K.; Yehl, K.; Spatz, J. P.; Cavalcanti-Adam, E. A.; Salaita, K. Nanoparticle Tension Probes Patterned at the Nanoscale: Impact of Integrin Clustering on Force Transmission. Nano Lett. 2014, 14, 5539-5546.

(35) Amschler, K.; Erpenbeck, L.; Kruss, S.; Schön, M. P. Nanoscale Integrin Ligand Patterns Determine Melanoma Cell Behavior. ACS Nano 2014, 8, 9113-9125.

(36) Amschler, K.; Kossmann, E.; Erpenbeck, L.; Kruss, S.; Schill, T.; Schön, M.; Möckel, S. M. C.; Spatz, J. P.; Schön, M. P. Nanoscale Tuning of VCAM-1 Determines VLA-4-Dependent Melanoma Cell Plasticity on RGD Motifs. Mol. Cancer Res. 2018, 16, 528-542.

(37) Dzamba, B. J.; Peters, D. M. Arrangement of Cellular Fibronectin in Noncollagenous Fibrils in Human Fibroblast Cultures. Journal of Cell Science 1991, 100 (3), 605-612.

(38) Leahy, D. J.; Aukhil, I.; Erickson, H. P. 2.0 Å Crystal Structure of a Four-Domain Segment of Human Fibronectin Encompassing the RGD Loop and Synergy Region. Cell 1996, 84, 155-164.

(39) Schiefner, A.; Gebauer, M.; Skerra, A. Extra-Domain B in Oncofetal Fibronectin Structurally Promotes Fibrillar Head-to-Tail Dimerization of Extracellular Matrix Protein. J. Biol. Chem. 2012, 287, 17578-17588.

(40) Früh, S. M.; Schoen, I.; Ries, J.; Vogel, V. Molecular Architecture of Native Fibronectin Fibrils. Nat. Commun. 2015, 6, 7275.

(41) Patla, I.; Volberg, T.; Elad, N.; Hirschfeld-Warneken, V.; Grashoff, C.; Fässler, R.; Spatz, J. P.; Geiger, B.; Medalia, O. Dissecting the Molecular Architecture of Integrin Adhesion Sites by Cryo-Electron Tomography. Nat. Cell Biol. 2010, 12, 909-915.

(42) Wallace, J. M.; Chen, Q.; Fang, M.; Erickson, B.; Orr, B. G.; Holl, M. M. B. Type I Collagen Exists as a Distribution of Nanoscale Morphologies in Teeth, Bones, and Tendons. Langmuir 2010, 26, $7349-7354$.

(43) Jiao, Y.; Feng, X.; Zhan, Y.; Wang, R.; Zheng, S.; Liu, W.; Zeng, $\mathrm{X}$. Matrix Metalloproteinase-2 Promotes $\alpha \mathrm{v} \beta 3$ Integrin-Mediated Adhesion and Migration of Human Melanoma Cells by Cleaving Fibronectin. PLoS One 2012, 7, No. e41591.

(44) Wang, K.; Wu, F.; Seo, B. R.; Fischbach, C.; Chen, W.; Hsu, L.; Gourdon, D. Breast Cancer Cells Alter the Dynamics of Stromal Fibronectin-Collagen Interactions. Matrix Biol. 2017, 60-61, 86-95. 
(45) Schaffner, F.; Ray, A.; Dontenwill, M. Integrin $\alpha 5 \beta 1$, the Fibronectin Receptor, as a Pertinent Therapeutic Target in Solid Tumors. Cancers 2013, 5, 27-47.

(46) Rechenmacher, F.; Neubauer, S.; Polleux, J.; Mas-Moruno, C.; De Simone, M.; Cavalcanti-Adam, E. A.; Spatz, J. P.; Fässler, R.; Kessler, H. Functionalizing $\alpha \mathrm{v} \beta 3$ - or $\alpha 5 \beta 1$-Selective Integrin Antagonists for Surface Coating: A Method to Discriminate Integrin Subtypes In Vitro. Angew. Chem., Int. Ed. 2013, 52, 1572-1575.

(47) Desoize, B.; Jardillier, J.-C. Multicellular Resistance: a Paradigm for Clinical Resistance? Critical Reviews in Oncology/Hematology 2000, 36, 193-207.

(48) Carragher, N. O.; Frame, M. C. Focal Adhesion and Actin Dynamics: A Place Where Kinases and Proteases Meet to Promote Invasion. Trends Cell Biol. 2004, 14, 241-249.

(49) Holle, A. W.; Engler, A. J. More Than a Feeling: Discovering, Understanding, and Influencing Mechanosensing Pathways. Curr. Opin. Biotechnol. 2011, 22, 648-654.

(50) Pelham, R. J.; Wang, Y. L. Cell Locomotion and Focal Adhesions Are Regulated by the Mechanical Properties of the Substrate. Biol. Bull. 1998, 194, 348-349 and discussion 349-350.

(51) Geiger, B.; Spatz, J. P.; Bershadsky, A. D. Environmental Sensing Through Focal Adhesions. Nat. Rev. Mol. Cell Biol. 2009, 10, 21-33.

(52) Glass, R.; Möller, M.; Spatz, J. P. Block Copolymer Micelle Nanolithography. Nanotechnology 2003, 14, 1153-1160.

(53) Schaufler, V.; Czichos-Medda, H.; Hirschfeld-Warnecken, V.; Neubauer, S.; Rechenmacher, F.; Medda, R.; Kessler, H.; Geiger, B.; Spatz, J. P.; Cavalcanti-Adam, E. A. Selective Binding and Lateral Clustering of $\alpha 5 \beta 1$ and $\alpha \mathrm{v} \beta 3$ Integrins: Unraveling the Spatial Requirements for Cell Spreading and Focal Adhesion Assembly. Cell Adhesion \& Migration 2016, 10, 505-515.

(54) Friedl, P.; Wolf, K. Tumour-Cell Invasion and Migration: Diversity and Escape Mechanisms. Nat. Rev. Cancer 2003, 3, 362374.

(55) Jung, J.-W.; Hwang, S.-Y.; Hwang, J.-S.; Oh, E.-S.; Park, S.; Han, I.-O. Ionising Radiation Induces Changes Associated with Epithelial-Mesenchymal Transdifferentiation and Increased Cell Motility of A549 Lung Epithelial Cells. Eur. J. Cancer 2007, 43, 1214-1224.

(56) Desgrosellier, J. S.; Cheresh, D. A. Integrins in Cancer: Biological Implications and Therapeutic Opportunities. Nat. Rev. Cancer 2010, 10, 9-22.

(57) Danen, E. H. J.; van Rheenen, J.; Franken, W.; Huveneers, S.; Sonneveld, P.; Jalink, K.; Sonnenberg, A. Integrins Control Motile Strategy Through a Rho-Cofilin Pathway. J. Cell Biol. 2005, 169, $515-526$.

(58) Taherian, A.; Li, X.; Liu, Y.; Haas, T. A. Differences in Integrin Expression and Signaling Within Human Breast Cancer Cells. BMC Cancer 2011, 11, 293.

(59) Bharadwaj, M.; Strohmeyer, N.; Colo, G. P.; Helenius, J.; Beerenwinkel, N.; Schiller, H. B.; Fässler, R.; Müller, D. J. $\alpha$ V-Class Integrins Exert Dual Roles on $\alpha 5 \beta 1$ Integrins to Strengthen Adhesion to Fibronectin. Nat. Commun. 2017, 8, 14348.

(60) Castelló-Cros, R.; Khan, D. R.; Simons, J.; Valianou, M.; Cukierman, E. Staged Stromal Extracellular 3D Matrices Differentially Regulate Breast Cancer Cell Responses Through PI3K and Beta1Integrins. BMC Cancer 2009, 9, 94.

(61) Zhao, F.; Li, L.; Guan, L.; Yang, H.; Wu, C.; Liu, Y. Roles for GP IIb/IIIa and $\alpha v \beta 3$ Integrins in MDA-MB-231 Cell Invasion and Shear Flow-Induced Cancer Cell Mechanotransduction. Cancer Lett. 2014, 344, 62-73.

(62) Zustiak, S.; Nossal, R.; Sackett, D. L. Multiwell Stiffness Assay for the Study of Cell Responsiveness to Cytotoxic Drugs. Biotechnol. Bioeng. 2014, 111, 396-403.

(63) Tokuda, E. Y.; Leight, J. L.; Anseth, K. S. Modulation of Matrix Elasticity with PEG Hydrogels to Study Melanoma Drug Responsiveness. Biomaterials 2014, 35, 4310-4318.

(64) Balcioglu, H. E.; van Hoorn, H.; Donato, D. M.; Schmidt, T.; Danen, E. H. J. The Integrin Expression Profile Modulates
Orientation and Dynamics of Force Transmission at Cell-Matrix Adhesions. J. Cell Sci. 2015, 128, 1316-1326.

(65) Miao, H.; Li, S.; Hu, Y.-L.; Yuan, S.; Zhao, Y.; Chen, B. P. C.; Puzon-McLaughlin, W.; Tarui, T.; Shyy, J. Y. J.; Takada, Y.; et al. Differential Regulation of Rho GTPases by $\beta 1$ and $\beta 3$ Integrins: the Role of an Extracellular Domain of Integrin in Intracellular Signaling. J. Cell Sci. 2002, 115, 2199-2206.

(66) Roca-Cusachs, P.; Gauthier, N. C.; del Rio, A.; Sheetz, M. P. Clustering of $\alpha 5 \beta 1$ Integrins Determines Adhesion Strength Whereas $\alpha \mathrm{v} \beta 3$ and Talin Enable Mechanotransduction. Proc. Natl. Acad. Sci. U. S. A. 2009, 106, 16245-16250.

(67) Roca-Cusachs, P.; Iskratsch, T.; Sheetz, M. P. Finding the Weakest Link - Exploring Integrin-Mediated Mechanical Molecular Pathways. J. Cell Sci. 2012, 125, 3025-3038.

(68) Graeter, S. V.; Huang, J.; Perschmann, N.; López-García, M.; Kessler, H.; Ding, J.; Spatz, J. P. Mimicking Cellular Environments by Nanostructured Soft Interfaces. Nano Lett. 2007, 7, 1413-1418. 